\title{
Estudio del comportamiento en enfriamiento continuo del acero martensítico ferrítico de activación reducida $\mathrm{F} 82 \mathrm{H}$
}

\author{
Study of the behavior of the reduced activation \\ martensitic ferritic steel F82H under \\ continuous cooling
}

Pablo Edgardo Reynoso Peitsch ${ }^{1}$, Claudio Ariel Danón ${ }^{1}$

\author{
${ }^{1}$ División Transformaciones de Fase - GM/CAC/GAEN/CNEA CP: B1650KNA, San Martín, Buenos Aires, Argentina. \\ e-mail: preynoso@cnea.gov.ar \\ e-mail: danon@cnea.gov.ar
}

\section{RESUMEN}

Los aceros martensítico-ferríticos $9 \% \mathrm{Cr}$ son, actualmente, candidatos privilegiados en la fabricación de componentes estructurales para los denominados reactores de fisión de Generación IV, debido a que presentan muy buenas propiedades termofísicas y mecánicas bajo irradiación de neutrones. Junto a éstos, los aceros martensítico-ferríticos de activación reducida, RAFM's por sus siglas en inglés -Reduced Activation Ferritic-

Martensitic Steels- son, del mismo modo, candidatos para los componentes estructurales en reactores de fusión. En esta contribución se presentan resultados relativos al comportamiento en transformación durante el enfriamiento continuo de un acero RAFM F82H, obtenidos al aplicar ciclos térmicos utilizando la técnica de Calorimetría Diferencial de Barrido (DSC). El estado de recepción del material utilizado fue normalizado a $1040{ }^{\circ} \mathrm{C}, 38^{\prime}$ y revenido a $750{ }^{\circ} \mathrm{C}, 60^{\prime}$. Se sometieron muestras a ciclos térmicos de tres segmentos: calentamiento a $5{ }^{\circ} \mathrm{C} / \mathrm{min}$ desde temperatura ambiente hasta la temperatura de austenizado, mantenimiento en fase austenítica durante 15 min a $1050{ }^{\circ} \mathrm{C}$ y enfriamiento a un conjunto de velocidades fijas, comprendidas entre 1,5 y $50{ }^{\circ} \mathrm{C} / \mathrm{min}$, manteniendo la presión constante. Luego de los ensayos de calorimetría, las muestras se prepararon por técnicas convencionales para su observación por microscopía óptica. Se implementó satisfactoriamente un algoritmo mediante el software Matlab que permite la determinación simultánea de la línea de base y la fracción transformada en función de la temperatura sobre la base de un procedimiento recursivo propuesto en la literatura previa. La transformación a la martensita en el acero F82H bajo las condiciones de ciclo térmico utilizadas en este trabajo mostró depender fuertemente de la velocidad de enfriamiento aplicada, produciéndose el desdoblamiento de la misma, o su ocurrencia en etapas diferenciadas, a medida que disminuye dicha velocidad.

Palabras clave: Aceros de activación reducida, transformación martensítica, calorimetría diferencial de barrido.

\section{ABSTRACT}

9\% Cr martensitic-ferritic steels are currently the privileged candidates to manufacture structural components of the so-called Generation IV nuclear fission reactors, because they exhibit very good thermophysical and mechanical properties under neutron irradiation. Along with them, the so-called Reduced Activation FerriticMartensitic steels (RAFM's) have been in the same way selected for the future nuclear fusion reactors. In this contribution we introduce results involving the transformation behavior under continuous cooling of the F82H RAFM steel, obtained by applying the differential scanning calorimetry (DSC) technique. The metallurgical state of the as-received material was normalized and tempered. The applied thermal cycles were as follows: heating at $5^{\circ} \mathrm{C} / \mathrm{min}$ up to $1050{ }^{\circ} \mathrm{C} / \mathrm{min}$ (austenite phase field), austenite holding for $15 \mathrm{~min}$. and cooling at fixed rates between 1.5 and $50{ }^{\circ} \mathrm{C} / \mathrm{min}$ under constant pressure. After DSC test, samples were prepared by conventional techniques to be observed by optical microscopy. An algorithm under Matlab language was satisfactorily developed which allowed the simultaneous determination of the baseline and the 
transformed fraction as a function of temperature on the basis of a recursive procedure proposed in the previous literature. The transformation to martensite for the F82H steel under the prescribed thermal cycle conditions showed to be strongly dependent on the applied cooling rate, giving rise to a splitting phenomenon -or a multi-step transformation mechanism- as the cooling rate is lowered.

Keywords: low activation steels, martensitic transformation, differential scanning calorimetry.

\section{INTRODUCCIÓN}

Desde su más temprano desarrollo, los aceros martensítico-ferríticos $9 \% \mathrm{Cr}$ han sido utilizados no solo en la fabricación de componentes de centrales térmicas, sino también en calderas, intercambiadores de calor, calentadores de alta presión, cañerías, tuberías, etc. debido a que poseen excelentes propiedades termofísicas y mecánicas, tales como resistencia al creep, tenacidad y resistencia a la oxidación a alta temperatura [1]. El mejoramiento continuo de las propiedades de materiales $9 \% \mathrm{Cr}$ ha permitido un incremento sustancial de sus prestaciones: aumento de las temperaturas de servicio -con el consiguiente aumento de la eficiencia- y obtención de valores cada vez más importantes de resistencia a la rotura. Hoy en día, en el ámbito de la generación de energía por medios nucleares, estos materiales son también candidatos privilegiados en la fabricación de componentes estructurales para los denominados reactores de fisión de Generación IV, debido a que presentan, además, buen comportamiento bajo irradiación de neutrones [2].

Asimismo, entre mediados de 1980 y finales de 1990 los materiales de alto Cr fueron introducidos en los programas internacionales de fusión nuclear bajo la forma de los denominados aceros martensíticoferríticos de activación reducida, RAFMs por sus siglas en inglés -Reduced Activation Ferritic-Martensitic Steels-, y luego de una extensa fase de evaluación de distintas composiciones de aleaciones Fe-Cr, se estableció un rango de composiciones 7-9\% Cr, convergiendo finalmente en composiciones similares a las aleaciones convencionales $9 \% \mathrm{Cr}[3]$.

En 1995, en el marco de esfuerzos conjuntos conducidos bajo la cobertura de la Agencia Internacional de Energía (IEA/Annex II) se define un acero de baja activación como referencia -el acero IEA-F82H: Fe$8 \% \mathrm{Cr}-2 \% \mathrm{~W}-\mathrm{TaV}$ - para comenzar a producirlo en Japón y, a continuación, ensayarlo. Una vez caracterizado el material por los participantes de IEA y luego de la validación de la información, se conformó una base de datos, que posteriormente fue utilizada para establecer los diseños de aleaciones F82H permitidos [4].

La literatura previa contiene estudios sobre aceros martensítico-ferríticos de tipo RAFM usando la técnica de calorimetría diferencial de barrido [5] y enfocados en aspectos tales como la cinética de la transformación martensítica, la energética de las transformaciones en ciclos de calentamiento y enfriamiento, etc., que aportan información relativa a los segmentos de calentamiento y enfriamiento continuo involucrados en cada caso.

En este trabajo estudiamos en particular el comportamiento del acero $\mathrm{F} 82 \mathrm{H}$ en ciclos térmicos de enfriamiento continuo para condiciones de austenización fijas, prestándose especial atención a la transformación a la martensita. Se presentan los resultados del comportamiento de la transformación obtenidos mediante el uso de las técnicas de microscopía óptica y electrónica de barrido de emisión por efecto de campo (FEGSEM) y calorimetría diferencial de barrido (DSC).

\section{MATERIALES Y MÉTODOS}

El material utilizado -a saber, una placa de $3 \mathrm{~mm}$ de espesor- se encontraba en el estado metalúrgico de recepción, esto es, normalizado a $1040^{\circ} \mathrm{C}, 38^{\prime}$ y revenido a $750{ }^{\circ} \mathrm{C}, 60^{\prime}$. Antes de extraer las muestras, ambas caras de la placa se desbastaron y pulieron con el fin de mejorar el contacto térmico entre las mismas y la base del crisol del DSC. Esta etapa implicó el uso de técnicas convencionales de preparación superficial para metalografía. A partir de la placa se obtuvieron entonces discos con un diámetro aproximado de $3.8 \mathrm{~mm}$ mediante corte por electroerosión, con un electrodo fabricado para tal fin. A su vez, se separó una muestra para el estudio metalográfico del material en estado de recepción.

En la Tabla 1 se muestra la composición química del material ensayado, acero RAFM F82H. 
Tabla 1: Composición química (\%p) del material.

\begin{tabular}{l|l|l|l}
\hline ELEMENTO & \%P & ELEMENTO & \%P \\
\hline $\mathrm{C}$ & 0,09 & $\mathrm{~S}$ & 0,002 \\
\hline $\mathrm{Cr}$ & 7,71 & $\mathrm{Ni}$ & 0,02 \\
\hline $\mathrm{Mo}$ & 0,003 & $\mathrm{~N}$ & 0,006 \\
\hline $\mathrm{W}$ & 1,95 & $\mathrm{Nb}$ & 0,0001 \\
\hline $\mathrm{Si}$ & 0,11 & $\mathrm{~V}$ & 0,16 \\
\hline $\mathrm{Mn}$ & 0,16 & $\mathrm{Ta}$ & 0,02 \\
\hline $\mathrm{P}$ & 0,002 & $\mathrm{Ti}$ & 0,01 \\
\hline
\end{tabular}

Las muestras obtenidas se pesaron en una balanza analítica, determinándose masas entre 75 y $90 \pm$ $0.01 \mathrm{mg}$. Las muestras se ensayaron en un calorímetro diferencial de barrido, marca Setaram, modelo LabSys Evo. Este equipo fue previamente calibrado en temperatura y flujo de calor utilizando las temperaturas y entalpías de fusión aceptadas en la literatura de los siguientes metales puros: In, Sn, Al, Ag, Fe. Las purezas de los mismos fueron de $99.999 \%$ para Sn y Ag, 99.997\% para In, 99.9999\% para Al y 99.98\% para Fe. [6] Para cada ensayo calorimétrico, se registró la temperatura del horno, la temperatura de la muestra y el flujo de calor en función del tiempo.

Se sometieron las muestras a ciclos térmicos que constaron de tres segmentos, a saber, un primer segmento de calentamiento desde temperatura ambiente hasta $1050{ }^{\circ} \mathrm{C}$ a una velocidad de $5{ }^{\circ} \mathrm{C} / \mathrm{min}$., un segundo de mantenimiento en el campo de fase austenítico y, por último, uno de enfriamiento a velocidad constante fijando diferentes velocidades, a saber: $1,5,2,3,5,10$ y $50{ }^{\circ} \mathrm{C} / \mathrm{min}$. Las experiencias se realizaron bajo atmósfera de argón con un caudal de $100 \mathrm{ml} / \mathrm{min}$, manteniendo la presión constante a $1 \mathrm{~atm}$.

Las velocidades de enfriamiento se seleccionaron de acuerdo a información previa [7,8] con el fin de intentar observar una o dos transformaciones durante el enfriamiento, a saber, transformación completa a la martensita o bien transformación incompleta a la ferrita, con su consecuente transición magnética (punto de Curie), seguida de transformación a la martensita (campo "bifásico").

Por otra parte, para descartar posibles efectos instrumentales en la evaluación de algunas anomalías observadas en los termogramas correspondientes a enfriamientos a $10,5,3$ y $2{ }^{\circ} \mathrm{C} / \mathrm{min}$, se llevaron a cabo, también, dichos ciclos térmicos en condición de ensayo "blanco", es decir, se reprodujeron los mismos ciclos térmicos sin muestra. Luego de los ensayos de calorimetría, las muestras (junto a la muestra en estado de recepción) se prepararon por técnicas convencionales para su observación por microscopía óptica y electrónica de barrido. El ataque químico para revelar la microestructura se hizo utilizando una mezcla de los reactivos Villela (50\%) y Nital 4\% (50\%). Las muestras se observaron con un microscopio óptico, marca Olympus, modelo GX51, perteneciente a la División Metalografía, Gerencia Materiales, CNEA y un microscopio electrónico de barrido de alta resolución con cañón de emisión por efecto de campo (FEG-SEM), marca Zeiss, modelo Supra 40, perteneciente al Centro de Microscopías Avanzadas de la Facultad de Ciencias Exactas y Naturales, Universidad de Buenos Aires.

Para procesar los datos experimentales obtenidos, correspondientes a las curvas flujo de calor vs. temperatura y flujo de calor vs. tiempo, se desarrolló un algoritmo utilizando el software Matlab que consta de 6 secciones principales, a saber: 1. aproximación lineal de los segmentos previo y posterior al pico calorimétrico; 2. obtención de las temperaturas de inicio y fin de transformación, utilizando el criterio de primera y segunda derivada; 3. obtención de las extrapolaciones lineales requeridas para el trazado de la línea de base por el método de BANDARA [9]; 4. obtención de la línea de base, utilizando el método de BANDARA [9]; 5. sustracción de la línea de base y obtención del calor de transformación; 6. obtención de la fracción transformada y su derivada temporal.

El método propuesto por BANDARA consiste en un algoritmo recursivo para la obtención de la línea de base, mediante la siguiente expresión matemática:

$F\left(t^{\prime}\right)=\alpha\left\{E_{2}\left(t^{\prime}\right)-E_{1}\left(t^{\prime}\right)\right\}+E_{1}\left(t^{\prime}\right)$

donde 


$$
\alpha=\frac{\int_{0}^{t^{\prime}}\{G(t)-F(t)\} d t}{\int_{0}^{t_{f}}\{G(t)-F(t)\} d t}
$$

es el grado de conversión (esto es, la fracción transformada); $G(t)$ es la curva experimental, $F(t)$ es la línea de base, $E_{i}(t)$ son las extrapolaciones de las porciones lineales anterior y posterior al pico calorimétrico y $t_{f}$ es el instante de finalización de la transición respectivamente.

\section{RESULTADOS}

\subsection{Microscopía}

En la Figura 1 se muestra la microestructura del material estudiado en el estado metalúrgico de recepción. Se observa inhomogeneidad en el tamaño del ex grano austenítico.

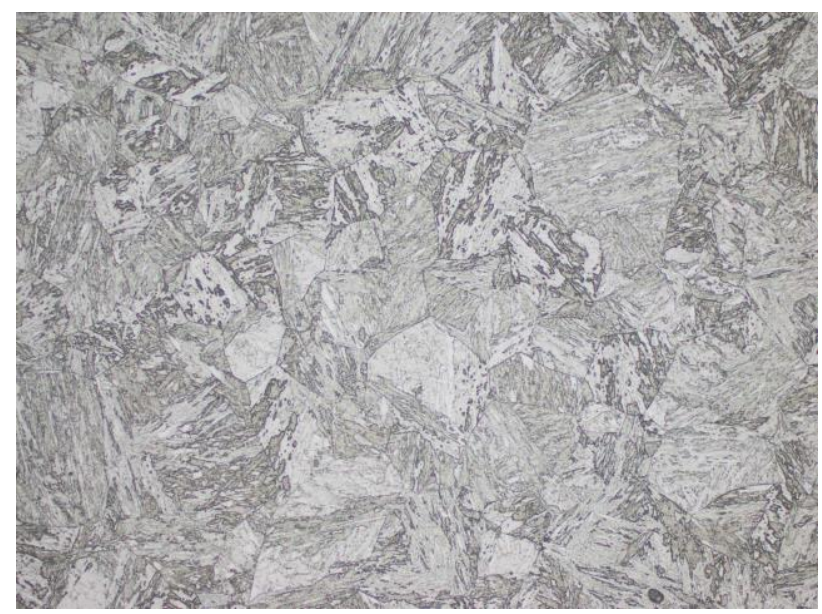

Figura 1: Microestructura del acero RAFM F82H en estado de recepción, normalizado a $1040{ }^{\circ} \mathrm{C}, 38^{\prime}$ y revenido a 750 ${ }^{\circ} \mathrm{C}, 60$ '.

En la Figura 2, (a), (b) y (c) se aprecia la microestructura obtenida para el acero F82H enfriando a 3, 2 y $1,5^{\circ} \mathrm{C} / \mathrm{min}$ respectivamente. Se distinguen claramente las fases ferrita, placas lisas y claras, y martensita, zonas oscuras menos definidas, con un aumento en la fracción de ferrita a medida que disminuye la velocidad de enfriamiento; para la velocidad de $1,5^{\circ} \mathrm{C} / \mathrm{min}$ la fracción de martensita presente en la muestra a temperatura ambiente disminuye notablemente, haciéndose prácticamente nula. Por su parte, las Figuras 3 y 4 muestran detalles de la microestructura obtenidos a partir de microscopia electrónica de barrido de emisión por efecto de campo para los ensayos realizados a 50 y $5^{\circ} \mathrm{C} / \mathrm{min}$ respectivamente. 

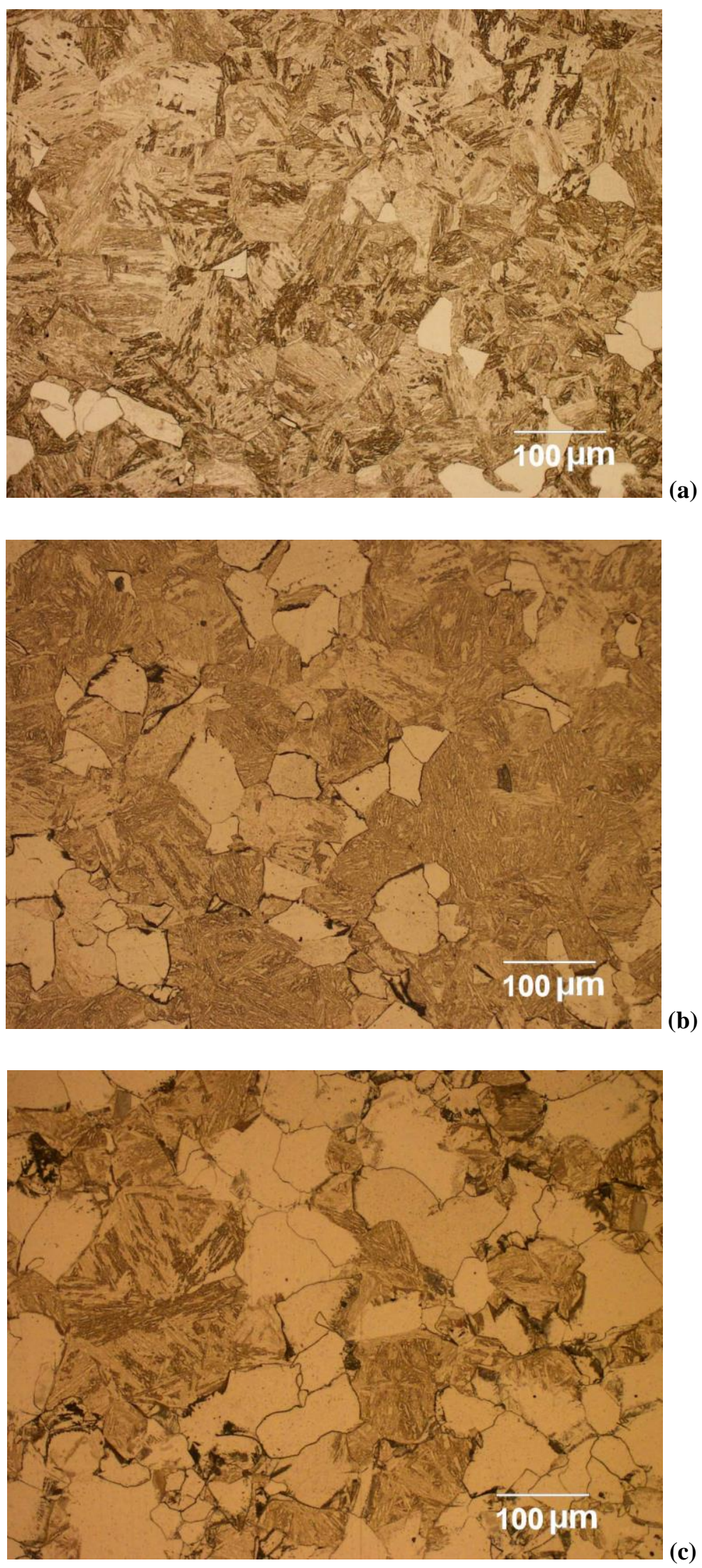

Figura 2: Microestructura del acero RAFM F82H enfriado a (a) $3{ }^{\circ} \mathrm{C} / \mathrm{min}$; (b) $2{ }^{\circ} \mathrm{C} / \mathrm{min}$, (c) $1,5^{\circ} \mathrm{C} / \mathrm{min}$. 

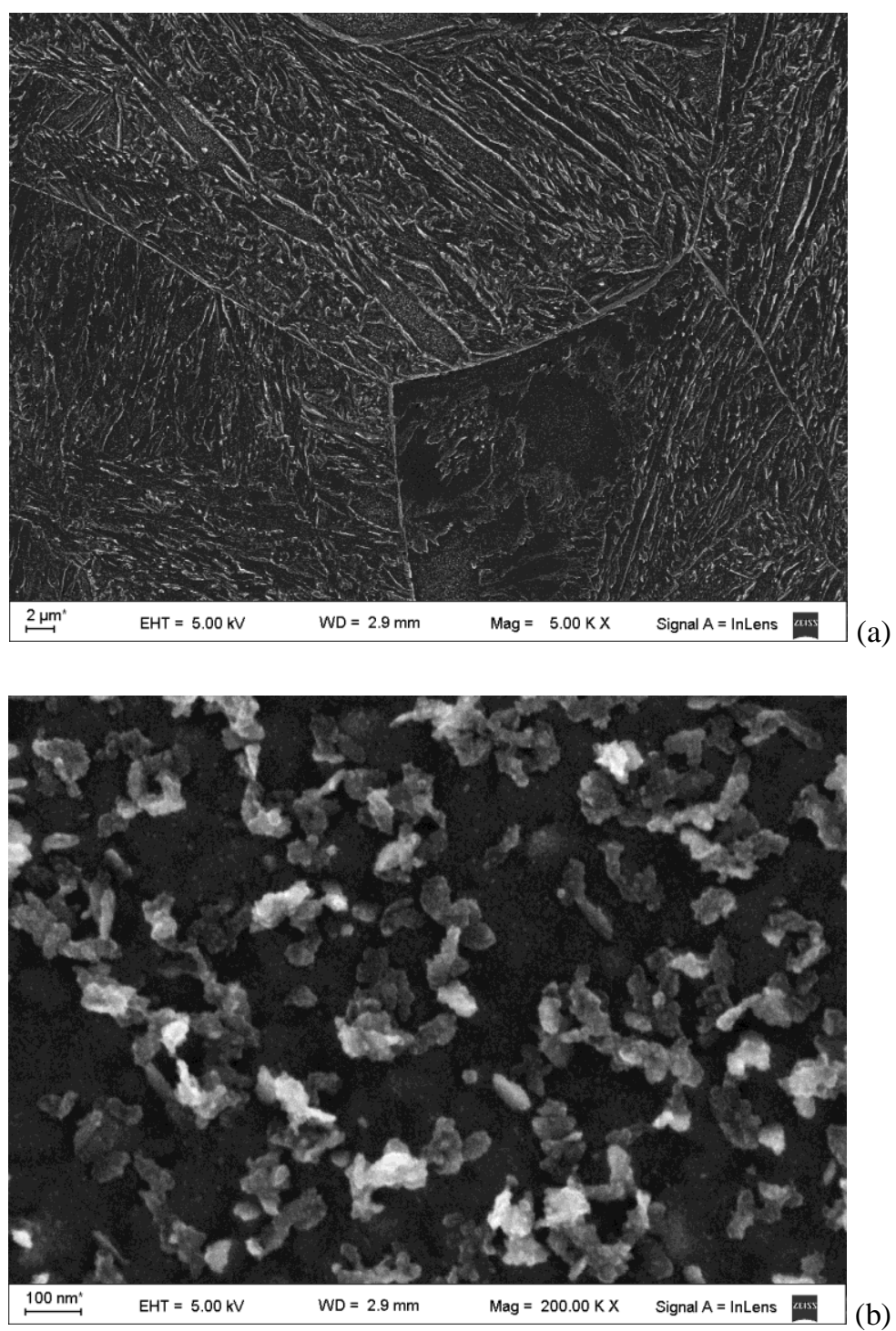

Figura 3: Microestructura del acero RAFM F82H enfriado a $50{ }^{\circ} \mathrm{C} / \mathrm{min}$ (FEG-SEM), (a) zona martensítica y (b) centro de la zona (a) con precipitados en listón martensítico.

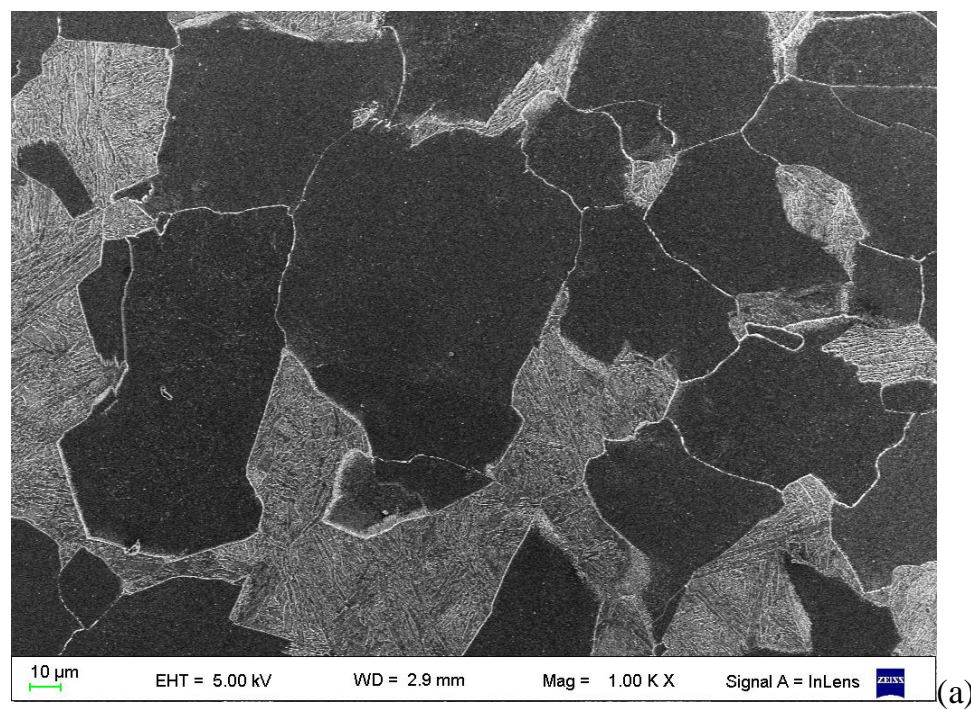




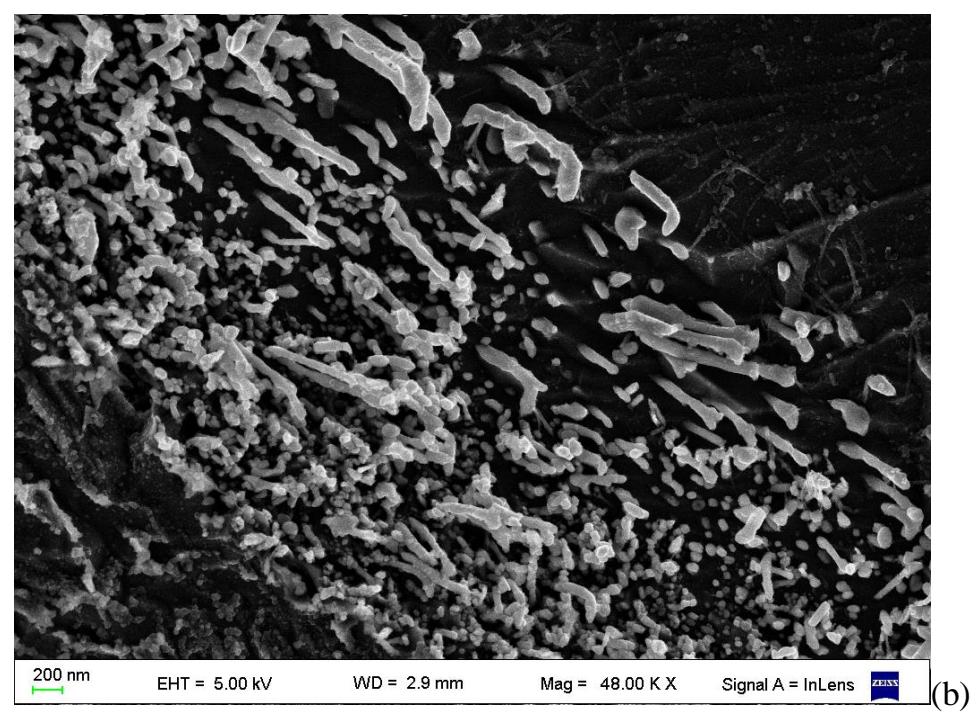

Figura 4: Microestructura del acero RAFM F82H enfriado a $5{ }^{\circ} \mathrm{C} / \mathrm{min}$ (FEG-SEM), (a) zona de dos fases: martensita en listones y placas ferríticas con precipitación en bordes, (b) detalle de la precipitación en el borde de las placas ferríticas.

De esta manera, las observaciones metalográficas sugieren que la velocidad crítica para la formación de ferrita en las condiciones experimentales especificadas se encuentra por debajo de $1,5^{\circ} \mathrm{C} / \mathrm{min}\left(90{ }^{\circ} \mathrm{C} / \mathrm{h}\right)$ para el acero estudiado. Esta estimación es similar a lo ya informado para ensayos realizados por las técnicas de dilatometría y calorimetría para el acero ASTM A335 P91 en trabajos anteriores realizados en nuestro laboratorio. La estimación de dicha velocidad arrojó valores entre 50 y $100{ }^{\circ} \mathrm{C} / \mathrm{h}$ [7] y por debajo de $90{ }^{\circ} \mathrm{C} / \mathrm{h}$ [8] utilizando técnicas de dilatometría y calorimetría respectivamente.

\subsection{Calorimetría diferencial de barrido: análisis cualitativo de la transformación austenita-martensita}

En la Figura 5 se muestra un detalle de un pico característico asociado a la transformación martensítica a una velocidad de enfriamiento de $50^{\circ} \mathrm{C} / \mathrm{min}$, en función del tiempo. Dicho pico se encuentra normalizado: se le aplicó un suavizado a los datos y se sustrajo la línea de base de acuerdo al procedimiento provisto por el software del instrumento. El método gráfico utilizado para obtener las temperaturas de transformación con esta metodología de tratamiento de datos ya se ha explicitado en un trabajo anterior [8].

En la Figura 6 se muestra un pico normalizado de igual forma que el pico en la Figura 5, asociado a la transformación martensítica de una muestra que sufrió un enfriamiento a una velocidad constante de $3^{\circ} \mathrm{C} / \mathrm{min}$. La comparación de las Figura 5 y 6 evidencia claramente una anomalía en su forma característica. Por otra parte, en la Figura 7 se exhiben en conjunto los segmentos de termogramas de enfriamiento continuo llevados a cabo para las distintas muestras a velocidades de 50,10, 5, 3, 2 y 1,5 ${ }^{\circ} \mathrm{C} / \mathrm{min}$, en función de la temperatura. Los datos de los segmentos de enfriamiento mencionados, en el rango de temperatura donde se evidencia la transformación a la martensita, fueron normalizados para poder ser graficados conjuntamente, luego de sustraer convenientemente la línea de base. En dichos segmentos se observa claramente el incremento del área del pico asociado a la transformación martensítica al aumentar la velocidad de enfriamiento. Esto evidencia, consistentemente con la observación por microscopia óptica y electrónica de barrido, un incremento de la fracción de austenita transformada a martensita. De igual manera, se hace evidente un cambio en la forma característica del pico mencionado a partir de velocidades de enfriamiento inferiores a $5^{\circ} \mathrm{C} / \mathrm{min}$. En efecto, en esta región de velocidades de enfriamiento $\left(\mathrm{v}_{\mathrm{E}}\right)$, el pico comienza primero a deformarse $\left(\mathrm{v}_{\mathrm{E}}=10 \mathrm{C} / \mathrm{min}\right) \mathrm{y}$ luego a desdoblarse, exhibiendo una serie de máximos y mínimos $\left(\mathrm{v}_{\mathrm{E}}=5,3,2\right.$ y $\left.1,5^{\circ} \mathrm{C} / \mathrm{min}\right)$. Se considera entonces, a partir de esta observación, la posibilidad de un desdoblamiento en la transformación martensítica, o bien una transformación por etapas. 


\section{F82H 1050gC 50x - $Q$ vs $t$, linea de base sustraida}

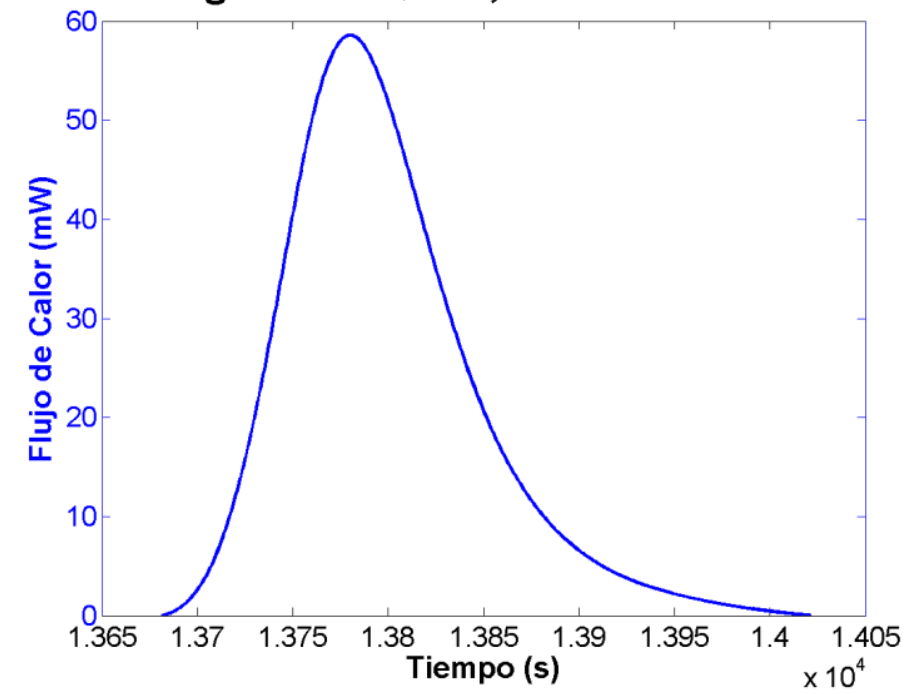

Figura 5: Pico asociado a la transformación martensítica normalizado (con suavizado de datos y sustracción de la línea de base) para el acero RAFM F82H enfriado a $50{ }^{\circ} \mathrm{C} / \mathrm{min}$.

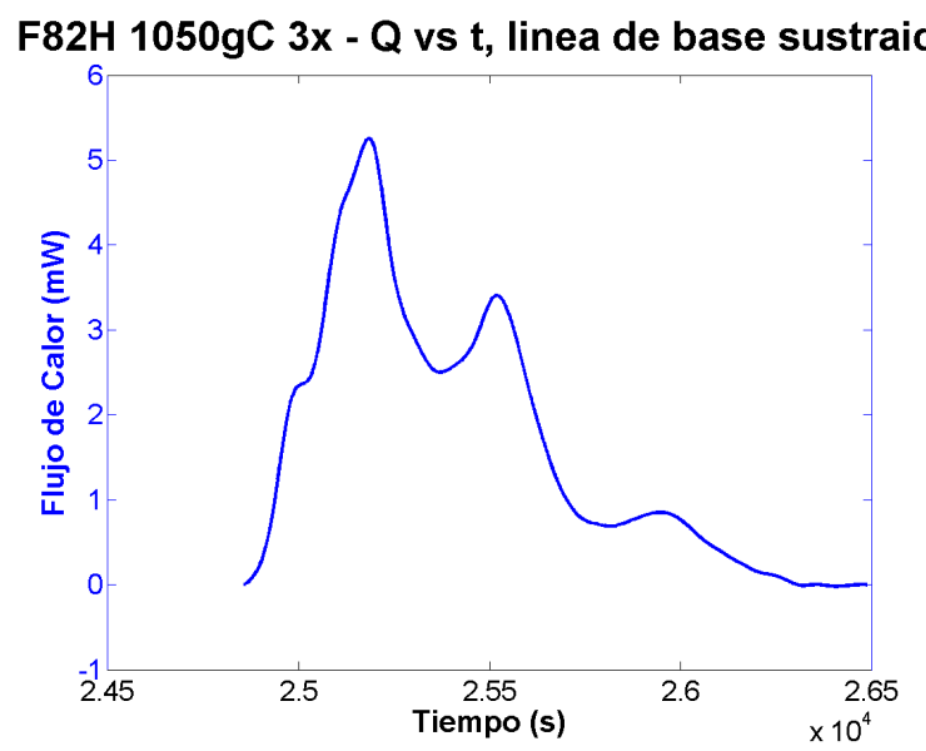

Figura 6: Conjunto anómalo de picos, asociado a la transformación martensítica de una muestra enfriada a $3^{\circ} \mathrm{C} / \mathrm{min}$ para el acero RAFM F82H. 


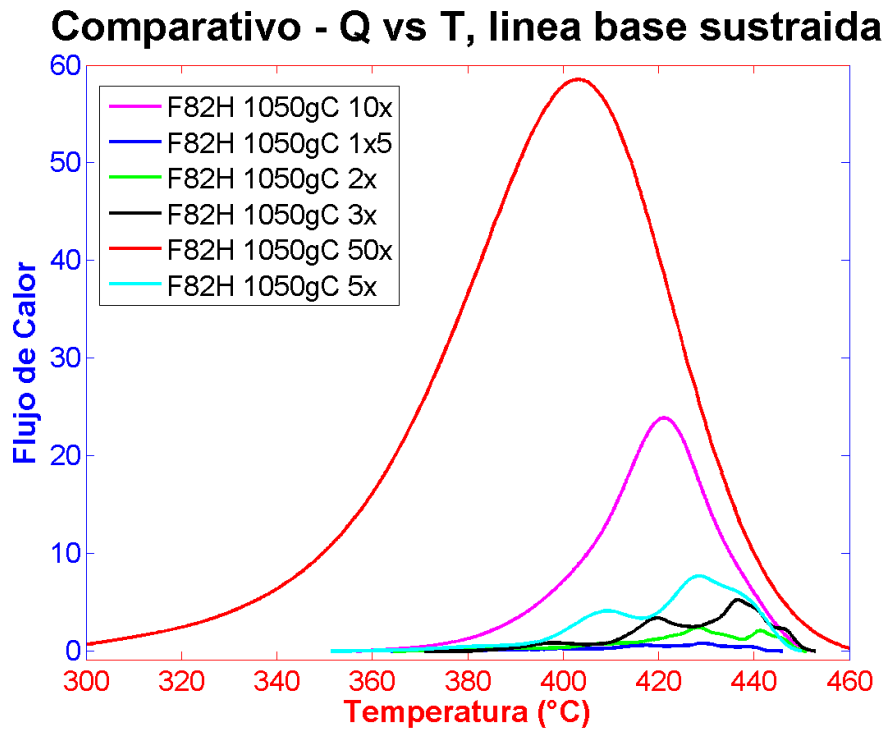

Figura 7: Comparación entre picos asociados a la transformación martensítica -con sustracción de la línea de base- en enfriamiento continuo a distintas velocidades, a saber 50, 10, 5, 3, 2 y $1,5^{\circ} \mathrm{C} / \mathrm{min}$, acero RAFM F82H.

\subsection{Calorimetría diferencial de barrido: análisis cuantitativo de la transformación austenita-martensita}

La Figura 8 ilustra las primeras etapas del método utilizado para la construcción de la línea de base (incluyendo la obtención de las temperaturas de comienzo y fin de transformación) mientras que la Figura 9 exhibe el resultado final del procedimiento recursivo aplicado siguiendo la propuesta de BANDARA [9].

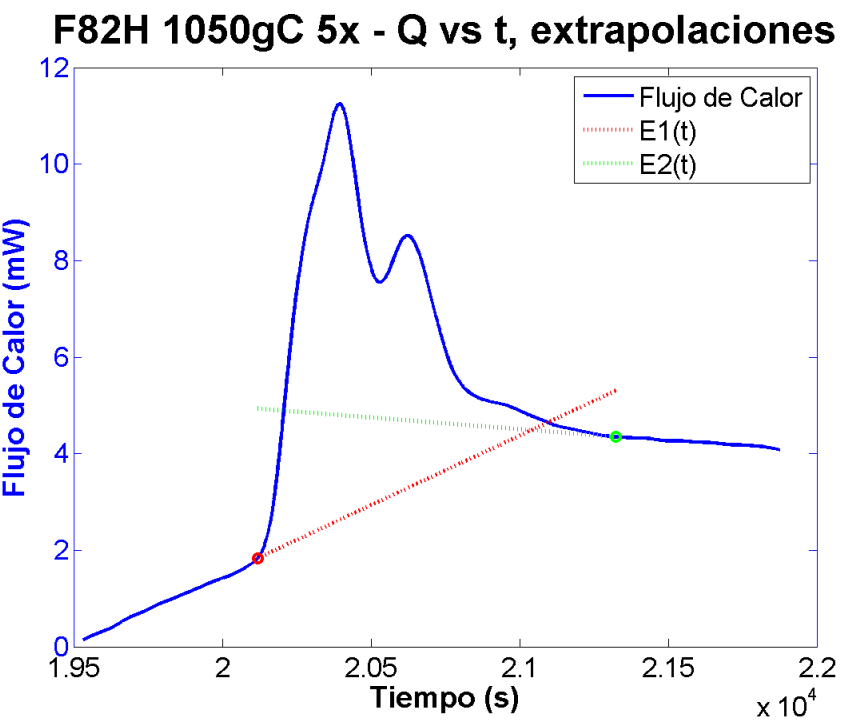

Figura 8: Extrapolaciones lineales, previa y posterior al pico asociado a la transformación martensítica, acero F82H enfriado a $5^{\circ} \mathrm{C} / \mathrm{min}$. Se muestran también las temperaturas de comienzo y fin de transformación. 


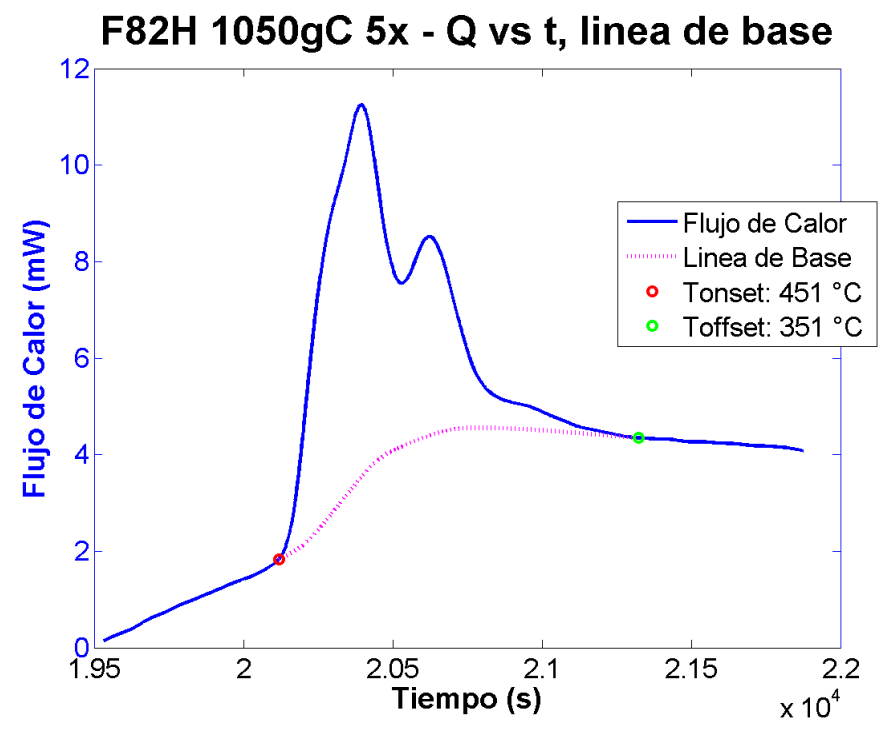

Figura 9: Pico asociado a la transformación martensítica con línea de base modelada según el método de BANDARA [9], acero F82H enfriado a $5^{\circ} \mathrm{C} / \mathrm{min}$.

Por otra parte, la Figura 10 muestra la fracción transformada a martensita en función de la temperatura, para cada una de las velocidades de enfriamiento ensayadas.

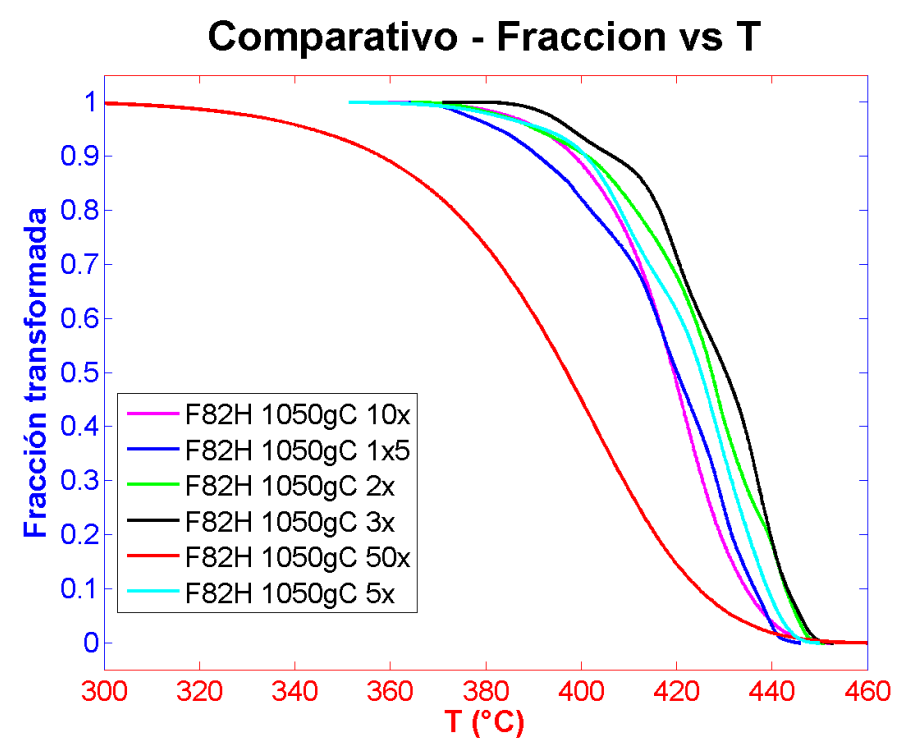

Figura 10: Curvas de fracción transformada de martensita para cada las velocidades de enfriamiento ensayadas, a saber $50,10,5,3,2$ y $1,5^{\circ} \mathrm{C} / \mathrm{min}$.

Finalmente, en la Tabla 2 se dan las temperaturas y calores de transformación a martensita para los diferentes ensayos calorimétricos. 
Tabla 2: Temperaturas y calores de transformación para la transición austenita-martensita a diferentes velocidades de enfriamiento, acero F82H

\begin{tabular}{l|l|l|l}
\hline $\mathbf{V}_{\text {ENF }}\left({ }^{\circ} \mathbf{C} / \mathbf{m i n}\right)$ & $\mathbf{T}_{\mathbf{1}}\left({ }^{\circ} \mathbf{C}\right)$ & $\mathbf{T}_{\mathbf{F}}\left({ }^{\circ} \mathbf{C}\right)$ & $\mathbf{Q}(\mathbf{m J})$ \\
\hline 50 & 465 & 296 & 6099 \\
\hline 10 & 453 & 357 & 4383 \\
\hline 5 & 451 & 351 & 2873 \\
\hline 3 & 453 & 380 & 2663 \\
\hline 2 & 450 & 369 & 1893 \\
\hline 1,5 & 447 & 365 & 1234 \\
\hline
\end{tabular}

\section{DISCUSIÓN}

El desdoblamiento o la ocurrencia en etapas de la transformación martensítica ha sido ya observado e informado en la literatura previa para diferentes tipos de aleaciones $[10,11,12]$ incluyendo los aceros de alto $\mathrm{Cr}$ $[13,14]$. Las hipótesis propuestas para explicar este fenómeno están enmarcadas en distintos protocolos experimentales y distintas composiciones químicas. Así, GARCÍA DE ANDRÉS et al. [12] apuntan a la transformación diferencial de distintas regiones de austenita, que coexisten con diferentes composiciones químicas debido a un insuficiente grado de homogeneización durante el ciclo térmico empleado. Otros autores, en cambio, sugieren que el fenómeno se produce por precipitación de carburos y/o crecimiento de carburos ya existentes, antes o después de producida la transformación [14]; dicha precipitación está, a su vez, asociada a la formación de listones anchos, que poseerían una temperatura de comienzo de formación de la martensita diferente de la correspondiente a los listones finos. Un tercer grupo de autores [11] postula que la modulación en la velocidad de transformación es plausiblemente consecuencia de la formación casi simultánea de bloques de listones de martensita dentro de todos los paquetes y en todos los granos, a lo largo del espécimen. Dicha simultaneidad aparecería a partir de condiciones de transformación localmente idénticas para la formación consecutiva de bloques adyacentes en un paquete. En fuerte contraste con esta última propuesta, se ha formulado también [10] la posibilidad de nucleación y crecimiento por pasos de regiones transformadas llamadas "cúmulos", a lo largo de un gran número de granos de austenita. La ocurrencia de una serie de máximos sucesivos en la velocidad de transformación se explicaría, dentro de este escenario, por el mecanismo de aceleración del crecimiento de cúmulos provisto por la formación autocatalítica de martensita, seguida de una desaceleración causada por las deformaciones por desajuste y las energías interfaciales entre las regiones transformadas y no transformadas.

En la evaluación de las diferentes hipótesis mencionadas arriba debe tenerse presente que las condiciones experimentales en nuestro caso difieren de las informadas en la literatura previa. Así, en las referencias [10] y [11] el enfriamiento se realiza a velocidad no sólo constante sino uniforme para todas las experiencias hasta llegar a una temperatura ligeramente superior a $\mathrm{M}_{\mathrm{s}}$, a partir de la cual sí se hace variar dicha velocidad. Esto asegura que la transformación a la martensita se realiza a partir de un estado metalúrgico "inicial"- en fase austenita- equivalente en todos los casos. En el presente trabajo se siguió un criterio diferente, a saber, se privilegió recabar información experimental sobre el diagrama de Transformación en Enfriamiento Continuo de la aleación bajo estudio, con la penalidad de obtener estados metalúrgicos "iniciales" (esto es, inmediatamente antes de la transformación a la martensita) diferentes para cada velocidad de enfriamiento. No obstante este hecho, tal como se anticipó en el comentario sobre la Figura 7, fue dable observar el fenómeno de desdoblamiento de la transformación estudiada independientemente de la fracción de fase transformada. En efecto, la transformación previa a la ferrita (bien sea por nucleación y crecimiento durante el enfriamiento, por exclusivo crecimiento de placas ferríticas ya nucleadas a alta temperatura, o por ambas razones) se manifiesta cuantitativamente en los resultados dados en la Tabla 2, en los que se aprecia la disminución del calor de transformación para la transición austenita-martensita con la velocidad de enfriamiento. Esta observación sugiere que el fenómeno de desdoblamiento es intrínseco a la transformación en sí y que su ocurrencia no depende del estado metalúrgico de la matriz previo al inicio de dicha transformación; en particular, de la fracción de austenita transformada a ferrita previamente.

Por otra parte, el valor de la temperatura $\mathrm{M}_{\mathrm{s}}$ no presentó una tendencia clara con la velocidad de en- 
friamiento entre los valores de 50 y $1,5^{\circ} \mathrm{C} / \mathrm{min}$. En la Tabla 2 se observa un valor significativamente mayor para la velocidad de $50^{\circ} \mathrm{C} / \mathrm{min}$ y luego una serie de valores con una ligera tendencia decreciente, que debería, sin embargo, ser corroborada. Se considera que la resolución de este punto requiere continuar el trabajo experimental con valores de velocidad de enfriamiento intermedios.

\section{CONCLUSIONES}

Los resultados y discusiones expuestos en las secciones anteriores dieron lugar a las siguientes conclusiones:

i. Los ensayos de comportamiento en enfriamiento continuo del acero martensítico-ferrítico de activación reducida F82H mostraron, en las condiciones experimentales disponibles, un aumento de la fracción en volumen de ferrita con la disminución de la velocidad de enfriamiento dentro del ámbito de velocidades ensayado. Las placas de ferrita exhibieron precipitación preferencial en los bordes. La velocidad crítica para la formación de ferrita se encuentra por debajo del valor $1.5^{\circ} \mathrm{C} / \mathrm{min}\left(90^{\circ} \mathrm{C} / \mathrm{h}\right)$.

ii. Se implementó satisfactoriamente un algoritmo mediante el software Matlab que permite la determinación simultánea de la línea de base y la fracción transformada austenita-martensita en función de la temperatura sobre la base de un procedimiento tomado de la literatura previa.

iii. La transformación a la martensita depende fuertemente de la velocidad de enfriamiento aplicada, produciéndose el desdoblamiento de la misma, o su ocurrencia en etapas diferenciadas, a medida que disminuye dicha velocidad.

\section{AGRADECIMIENTOS}

Este trabajo fue parcialmente financiado con aportes del Proyecto de Investigación Científica y Tecnológica 2170 -2014 del Foncyt-Mincyt (Argentina).

\section{BIBLIOGRAFÍA}

[1] KLUEH, R.L., NELSON, A.T., "Ferritic/martensitic steels for next-generation reactors", Journal of $\mathrm{Nu}$ clear Materials, v. 371, n. 1-3, pp. 37-52, Sep. 2007.

[2] VAN DER SCHAAF, B., GELLES, D.S, JITSUKAWA, S., et al., "Progress and critical issues of reduced activation ferritic/martensitic steel development", Journal of Nuclear Materials, v. 283-287(Part 1), pp. 52-59, Dec. 2000.

[3] KOHYAMA, A., HISHINUMA, A, GELLES, D.S., et al., "Low-activation ferritic and martensitic steels for fusion application", Journal of Nuclear Materials, v. 233-237(Part 1), pp. 138- 147, Oct. 1996.

[4] TAVASSOLI, A.A.F., DIEGELE, E., et al., "Current status and recent research achievements in ferritic/martensitic steels", Journal of Nuclear Materials, v. 455, n. 1-3, pp. 269-276, Dec. 2014.

[5] RAJU, S., JEYA GANESH, B., KUMAR RAI, A., et al., "A study on martensitic phase transformation in $9 \mathrm{Cr}-1 \mathrm{~W}-0.23 \mathrm{~V}-0.063 \mathrm{Ta}-0.56 \mathrm{Mn}-0.09 \mathrm{C}-0.02 \mathrm{~N}$ (wt.\%) reduced activation steel using differential scanning calorimetry", Journal of Nuclear Materials, v. 405, n.1, pp. 59-69, Oct. 2010.

[6] REYNOSO PEITSCH, P., DANON, C.A., "Procedimiento de calibración en temperatura y flujo de calor de un calorímetro diferencial de barrido (DSC)", Avances en Ciencia e Ingeniería, v. 7, n. 4, pp. 17-27, 2016.

[7] CARRIZO, D.A., "Comportamiento en transformación y caracterización microestructural de aceros de alta temperatura para reactores nucleares avanzados", Tesis de Maestría en Ciencia y Tecnología de Materiales, Instituto Sábato, UNSAM-CNEA, San Martín, Buenos Aires, 2012.

[8] REYNOSO PEITSCH, P., DANON, C.A., "Comparative Study of 9\% Cr Martensitic-ferritic Steels Using Differential Scanning Calorimetry”, Procedia Materials Science, v. 9, pp. 514-522, 2015.

[9] BANDARA, U., "A systematic solution to the problem of sample background correction in DSC curves", Journal of Thermal Analysis, v. 31, pp.1063-1071, 1986.

[10] VILLA, M., PANTLEON K., REICH, M., et al., Sommers, M.A.J., "Kinetics of anomalous multi-step formation of lath martensite in steel", Acta Materialia, v. 80, pp. 468-477, Nov. 2014.

[11] LOEWY, S., RHEINGANS, B., RAMUDU MEKA, S., et al., "Unusual martensite-formation kinetics in steels: observation of discontinuous transformation rates", Acta Materialia, v. 64, pp. 93-99, Feb. 2014.

[12] GARCÍA DE ANDRÉS, C., JIMÉNEZ, J.A., ALVAREZ, L. F. "Splitting phenomena occurring in the martensitic transformation of $\mathrm{Cr} 13$ and CrMoV14 stainless steels in the absence of carbide precipitation", 
Metallurgical and Materials Transactions, v. 27A, n. 7, pp.1799-1805, Jul. 1996.

[13] TAO, X., HAN, L., GU, J., "Splitting phenomenon in martensitic transformation of X12CrMoWVNbN10-1-1 steel”, International Journal of Materials Research, v. 106, n. 6, pp. 565-571, Jun. 2015.

[14] LIU, C., LIU, Y., ZHANG, D., et al., "Research on splitting phenomenon of isochronal martensitic transformation in T91 ferritic steel”, Phase Transitions, v. 85, n. 5, pp. 461-470, 2012. 